\title{
A case report of ventricular dysfunction post pericardiocentesis: stress cardiomyopathy or pericardial decompression syndrome?
}

\author{
Chadi Ayoub ${ }^{1,2+}$, Michael Chang ${ }^{1,2+}$ and Leonard Kritharides ${ }^{1,2^{*}}$
}

\begin{abstract}
We report a case of transient biventricular dysfunction post therapeutic pericardiocentesis, with classic features of stress cardiomyopathy (SCM). In our patient, the clinical and echocardiographic features were more in keeping with Takotsubo-type SCM than pericardial decompression syndrome (PDS). Our case is instructive in challenging our understanding of the aetiology of LV dysfunction complicating pericardiocentesis, and in highlighting the importance of careful clinical evaluation (altered heart rate and dyspnoea) in suspecting acute LV dysfunction after initial clinical improvement with pericardial aspiration.
\end{abstract}

Keywords: Pericardiocentesis, Stress cardiomyopathy, Pericardial decompression syndrome, Ventricular dysfunction, Echocardiography

\section{Background}

We report a case of reversible biventricular dysfunction following successful pericardiocentesis with classic features of stress or "Takotsubo" cardiomyopathy (SCM). Reports of SCM after pericardiocentesis are rare [1], as distinct from so-called pericardial decompression syndrome (PDS) which encompasses a spectrum of features of cardiac decompensation after large volume pericardiocentesis, including pulmonary oedema, adult respiratory distress syndrome, severe bi-ventricular failure and cardiogenic shock [2]. Our case is instructive in challenging our understanding of the aetiology of LV dysfunction complicating pericardiocentesis, and in highlighting the importance of careful clinical observations (heart rate and dyspnoea) in suspecting acute LV dysfunction after initial clinical improvement with pericardiocentesis.

\section{Case report}

A 62-year-old male presented with progressive dyspnoea for 10 days. He had a background of stage IV metastatic

\footnotetext{
* Correspondence: leonard.kritharides@sydney.edu.au

${ }^{\dagger}$ Equal contributors

'Department of Cardiology, Concord Repatriation General Hospital, Concord 2139, NSW, Australia

${ }^{2}$ The University of Sydney, Sydney, NSW, Australia
}

non-small lung carcinoma treated for 6 months with non-cardiotoxic chemotherapy (carboplatin and gemcitabine), and recently commenced on target therapy (Erlotinib). Clinical examination revealed signs consistent with cardiac tamponade, including significant pulsus paradoxus, tachycardia (heart rate 101), tachypnea (respiratory rate 25), elevated jugular venous pressure and muffled heart sounds. He was normotensive at 130/90mmHg. The patient was extremely anxious and spontaneously expressed concern about his imminent death.

His electrocardiogram (ECG) (Fig. 1) demonstrated electrical alternans and bedside transthoracic echocardiography (TTE) revealed a large pericardial effusion with features of cardiac tamponade, including diastolic compression of both right atrium and ventricle (Fig. 2, Additional file 1: Video 1 and Additional file 2: Video 2) and large mitral inflow variation (Fig. 3). Urgent pericardiocentesis was performed with a restricted aspiration of only $600 \mathrm{ml}$ drained initially over the first hour, and a total drainage of $1.8 \mathrm{l}$ of heavily blood-stained pericardial fluid over $36 \mathrm{~h}$. During initial aspiration of pericardial fluid there was immediate symptomatic relief and haemodynamic improvement (heart rate [HR] decreased to $80 / \mathrm{min}$, respiratory rate [RR] decreased to 15 breaths/min and BP increased to $150 / 70 \mathrm{mmHg}$ ). 


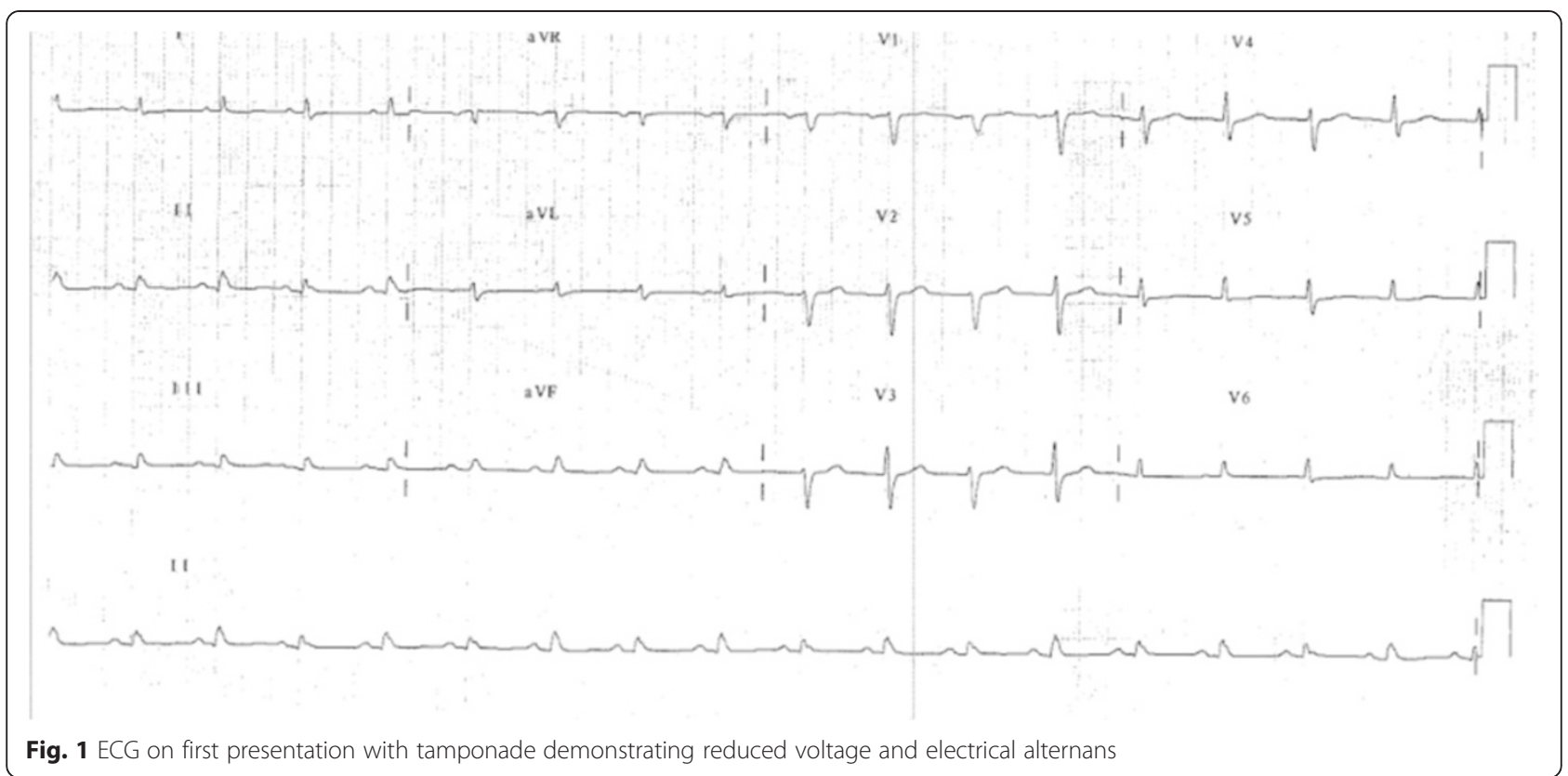

Overnight ( $9 \mathrm{~h}$ post procedure) the patient developed chest discomfort, dyspnea, tachycardia (HR 110) and tachypnoea (RR 24). TTE the next morning showed no re-accumulation of pericardial fluid, but detected new severe impairment in function of both ventricles, with akinesis of the apex and peri-apical region (Figs. 4 and 5, Additional file 3: Video 3 and Additional file 4: Video 4). Biomarkers demonstrated a rise in highly sensitive troponin from 8 to 224ng/L, but creatinine kinase did not rise significantly (107 to $116 \mathrm{U} / \mathrm{L}$ ). ECG after chest pain demonstrated resolution of the electrical alternans, with new loss of $\mathrm{R}$ waves in the anterior leads (Fig. 6).

Based on a presumptive diagnosis of SCM, angiotensin converting enzyme inhibitor and long acting betablocker were commenced, chemotherapy withheld and the patient discharged for early clinical and echocardiographic review. Serial follow up TTEs showed normalization of bi-ventricular function after two weeks (Figs. 7 and 8, Additional file 5: Video 5 and Additional file 6: Video 6), and restoration of $\mathrm{R}$ waves on subsequent

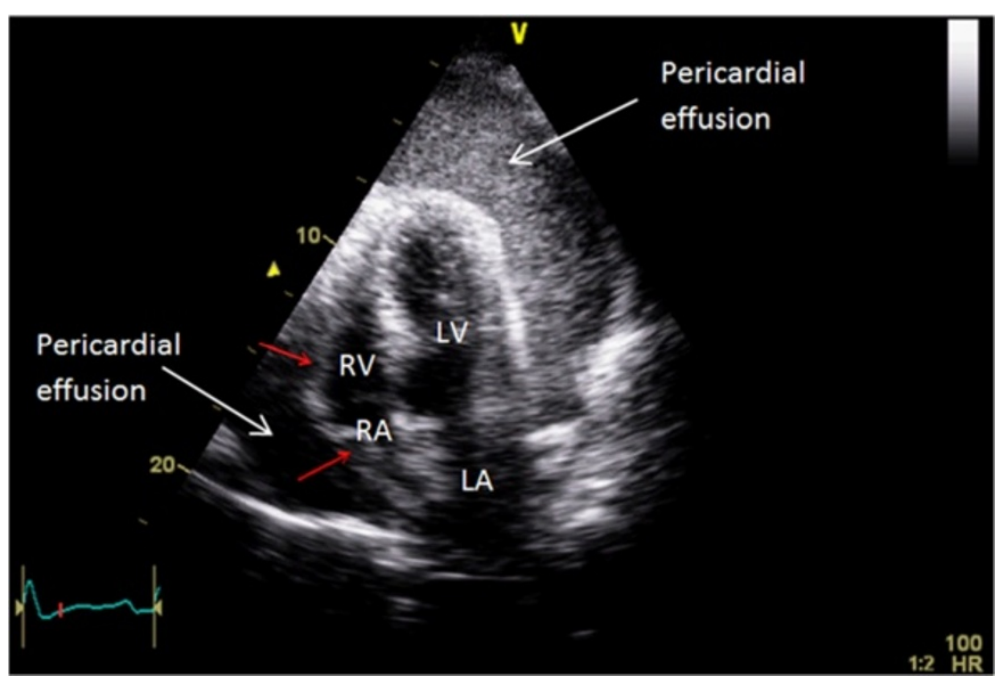

Fig. 2 Apical four chamber view on initial presenation demonstating large pericardial effusion with tamponade causing compression of right heart chambers (red arrows). LV function was normal prior to pericardiocentesis 


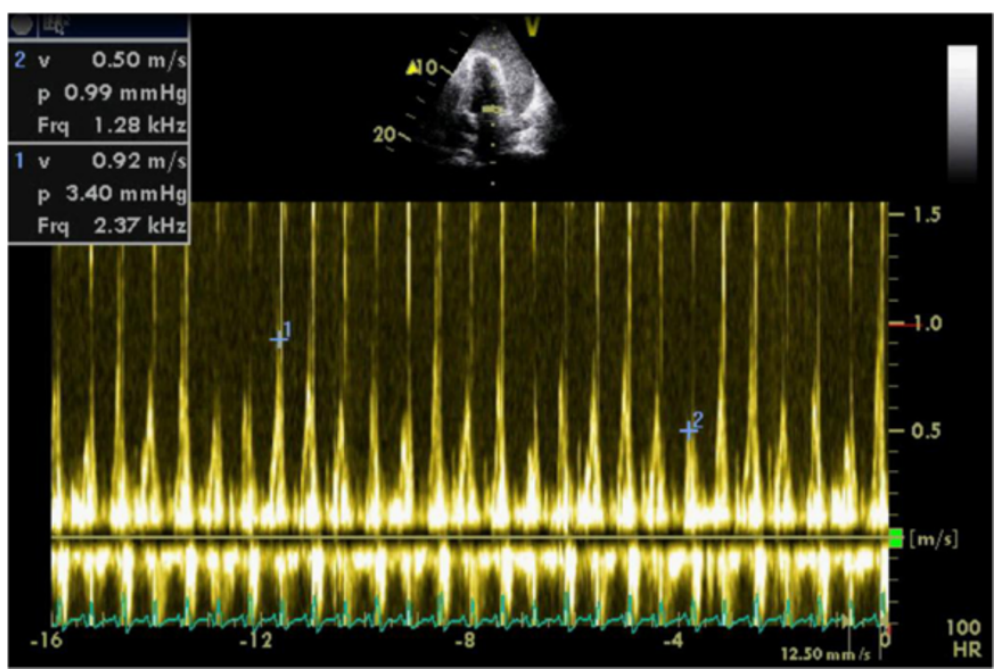

Fig. 3 Transmitral inflow traces showing signicicant respiratory phase variation, consistant with tamponade on first presentation

ECGs (Fig. 9). Subsequent computed tomography examination showed normal coronary arteries with a calcium score of zero and no evidence of LAD laceration or dissection.

The patient presented three months later with reaccumulation of pericardial effusion and tamponade. Therapeutic pericardiocentesis was performed with $500 \mathrm{ml}$ of blood stained pericardial fluid drained immediately, with $1.9 \mathrm{~L}$ in total over $36 \mathrm{~h}$. On this presentation he was relaxed and well adjusted in regards to his diagnosis. No LV dysfunction was detected on serial follow-up echocardiograms after the second pericardiocentesis (Fig. 10).

\section{Discussion}

Our patient developed biventricular apical dysfunction following successful and judicious pericardiocentesis, with features typical of stress or "Takotsubo cardiomyopathy". The case is instructive for its comparison with PDS and the clinical pattern of initial improvement followed by deterioration respectively due to pericardial aspiration and myocardial pathology.

In light of the timing of onset of biventricular impairment immediately post procedure PDS is an important differential diagnosis. Other differentials such as laceration to the ventricle or left anterior descending (LAD) coronary artery were clinically unlikely. The former was

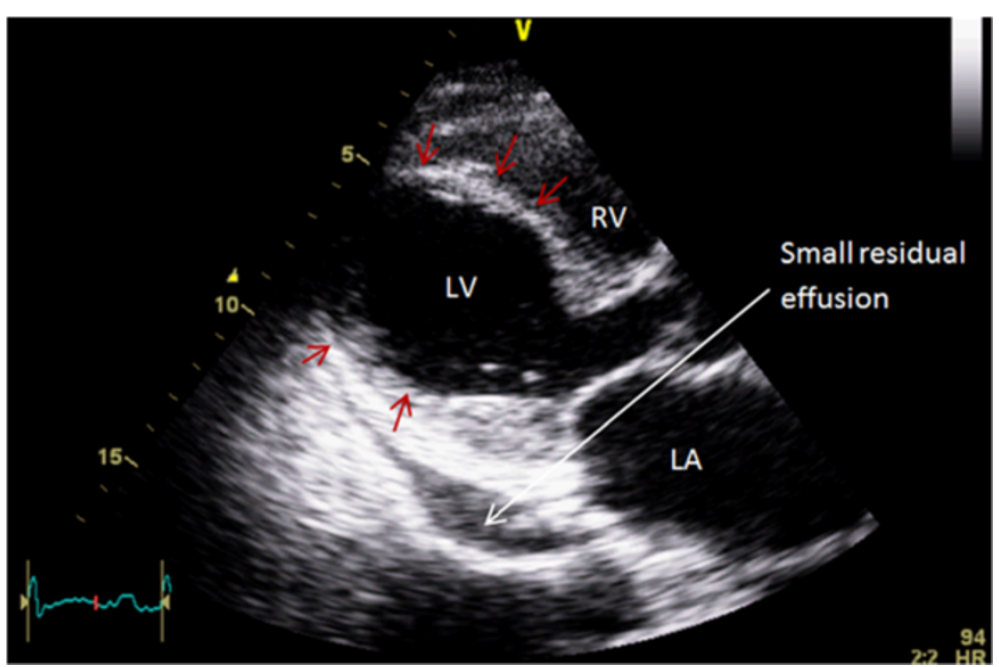

Fig. 4 Parasternal long view post pericardiocentesis demonstrating apical ballooning (red arrows) as a result of apical and peri-apical akinesis 


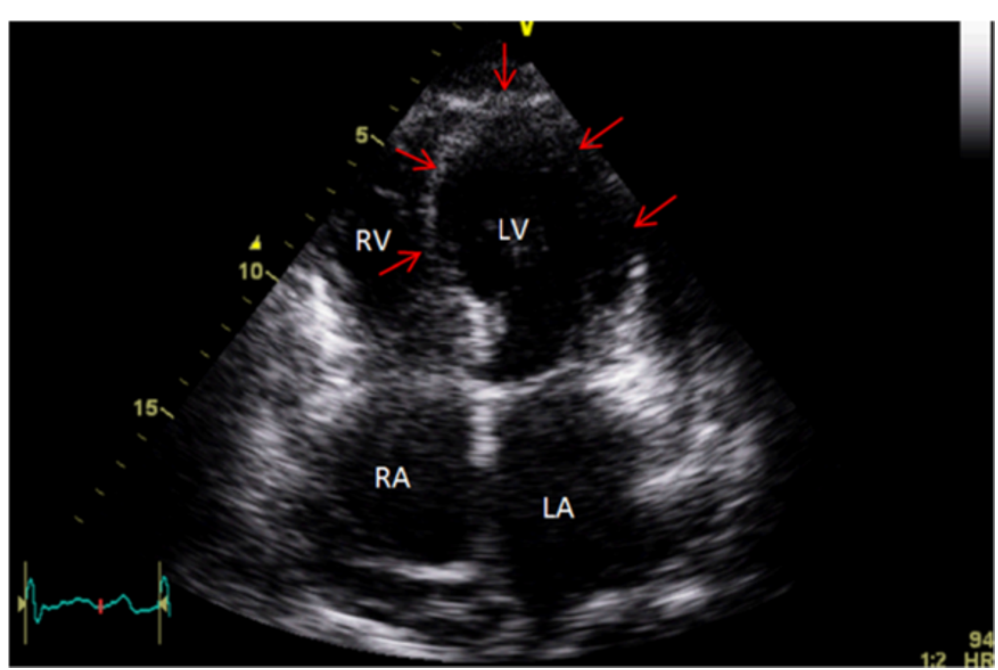

Fig. 5 Apical four chamber view post pericardiocentesis demonstrating apical ballooning (red arrows) as a result of apical and peri-apical akinesis

excluded by the absence of new pericardial bleed post procedure. Laceration of the LAD was also clinically unlikely given relatively small rise in cardiac enzymes and absence of large infarct, the presence of concurrent RV dysfunction, spontaneous recovery of ventricular function in a short period of time; additionally CT scan showed no evidence of haematoma or injury to the LAD.

Accordingly, we reviewed the literature describing SCM and PDS. Whereas SCM has been rarely reported after pericardiocentesis, much has been published on
PDS. The incidence of PDS or new left or right systolic dysfunction has been reported to range from $5 \%$ to $36 \%$ of patients post pericardiocentesis [3, 4], especially after malignant pericardial effusions. Although the first case report of PDS in 1983 noted APO with preserved LV function [5], most subsequent reports describe severe impairment of left, right or bi- ventricular function, which may be segmental or global (Tables 1 and 2).

A number of mechanisms have been proposed to explain the pathogenesis of LV systolic dysfunction in PDS.

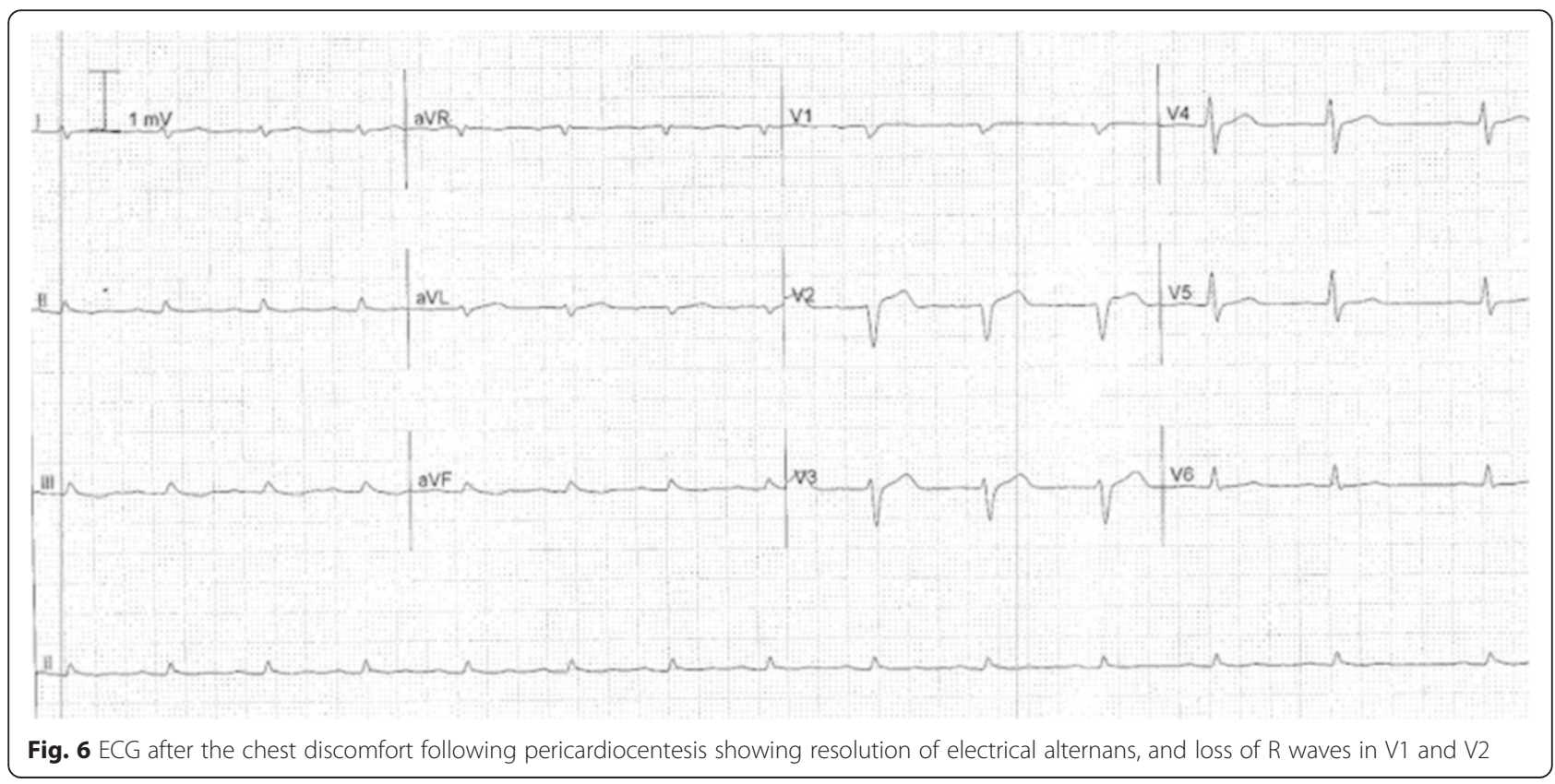




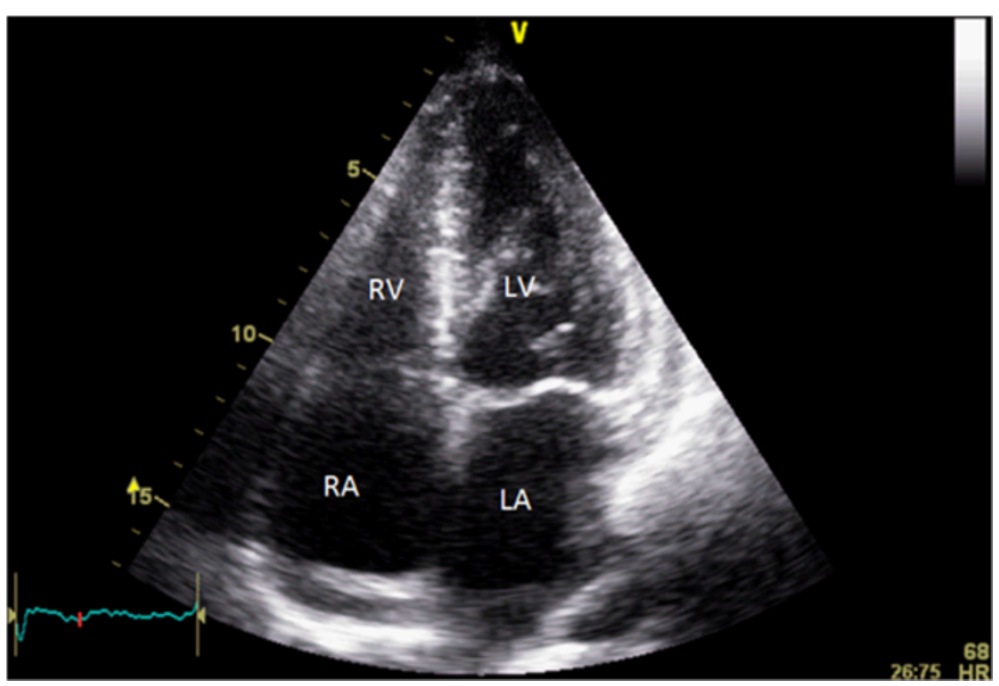

Fig. 7 Is an apical four chamber view 2 weeks post pericardiocentesis and development of LV dysfunction showing resolution of the apical ballooning in systole with normal LV systolic function

Acute withdrawal of exaggerated sympathetic drive during relief of tamponade may trigger paradoxical haemodynamic instability [5]. Mechanical, inter-ventricular volume mismatch may also contribute, with sudden relief of pericardial constraint leading to abrupt, disproportionate increase in RV volume and a paradoxical rise in pulmonary artery pressure, resulting in raised LV end diastolic pressure and transient left heart failure [5-9]. Others have proposed myocardial stunning from coronary perfusion mismatch with acute distension of cardiac chambers after decompression $[6,10,11]$. Taken together, it is likely that a combination of hormonal and mechanical pathophysiologic mechanisms contribute to LV dysfunction and the final clinical sequelae in PDS.

The classic echocardiographic feature in SCM is transient LV apical ballooning, although other segmental patterns have been described [12, 13]. A stressor leading to sympathetic overdrive and excessive catecholamine release is the currently accepted trigger in the development of SCM [12]. The catecholamine surge precipitates 1) 'peripheral arterial vasospasm leading to increased afterload and transient increase in LV end-systolic pressure, 2) 'acute multiple coronary artery vasospasm leading to myocardial ischaemia, and 3) direct catecholamine-

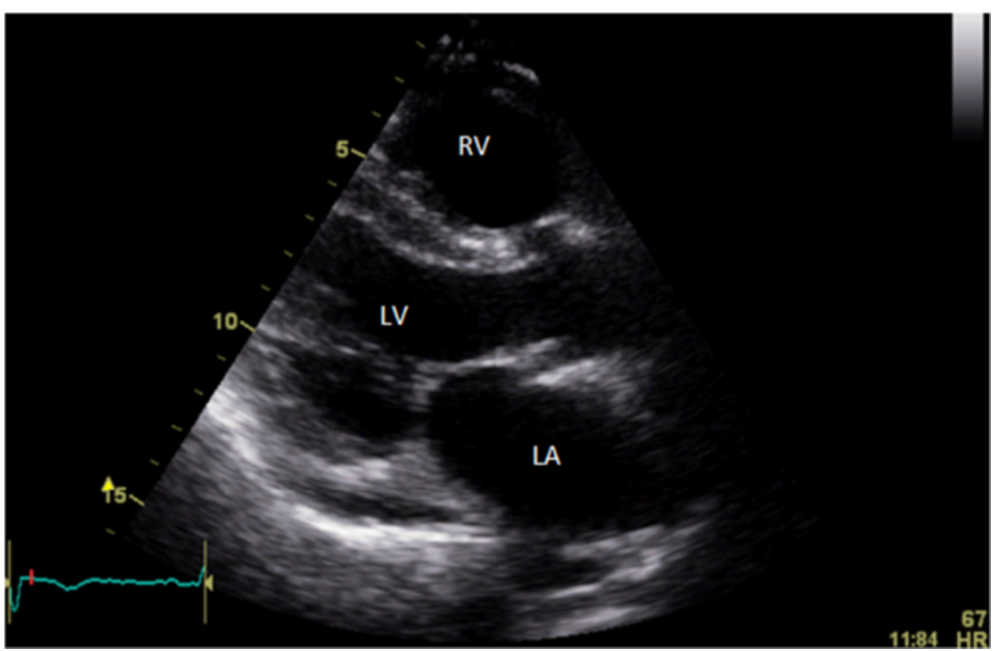

Fig. 8 Parasternal long view in systole (2 weeks post pericardiocentesis and development of LV dysfunction), showing resolution of both akinesis in the mid septum and apical ballooning (apex not well visulised here) 

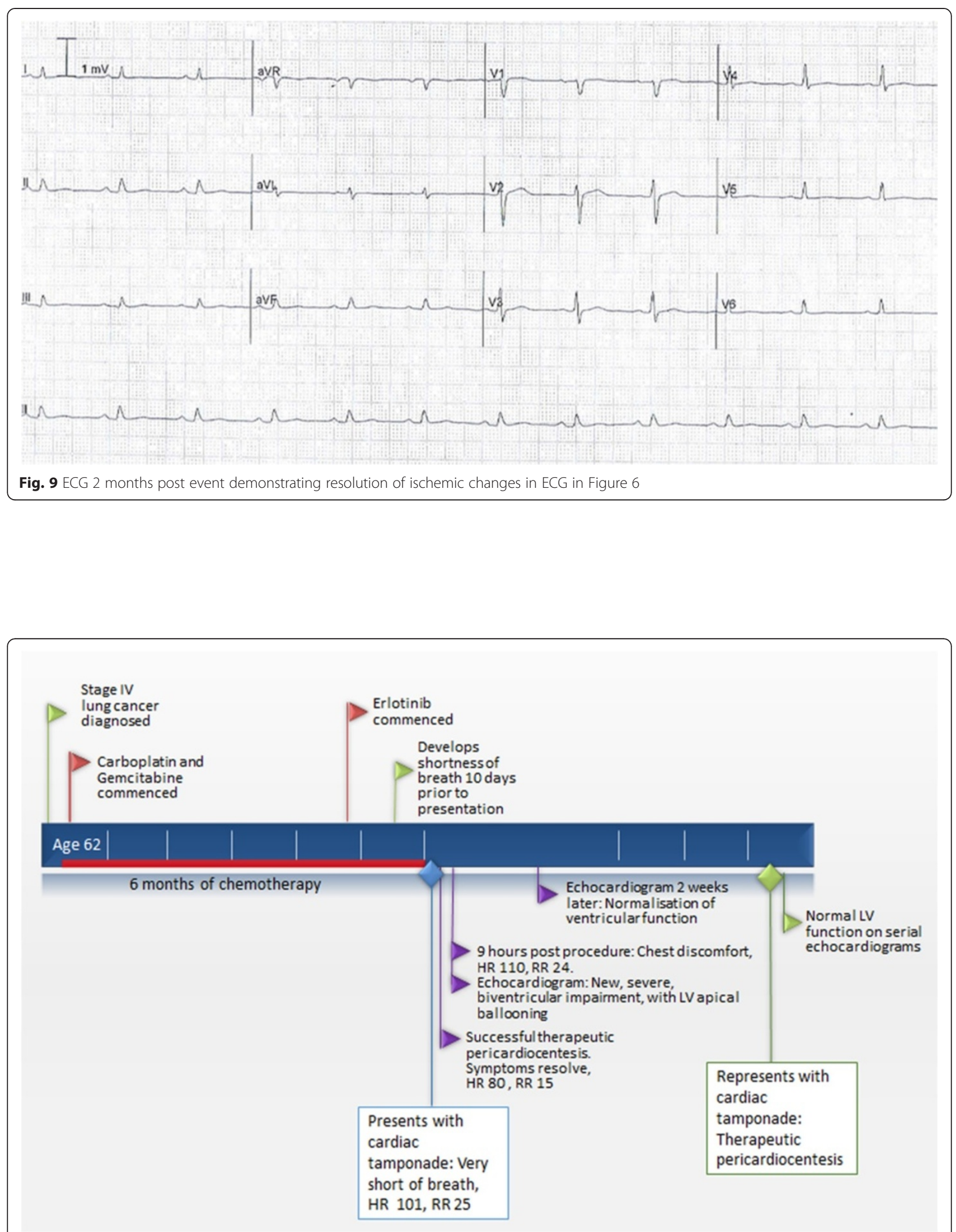

Fig. 10 Time line of clinical events 
Table 1 Summary of reported cases of LVF post pericardiocentesis: Clinical characteristics

\begin{tabular}{|c|c|c|c|c|c|c|c|c|c|}
\hline Report & $\begin{array}{l}\text { Age/ } \\
\text { Gender }\end{array}$ & Clinical Scenario & $\begin{array}{l}\text { Chronicity } \\
\text { of effusion }\end{array}$ & Type of pericardi-ocentesis & Nature of pericardial fluid & Fluid drained & $\begin{array}{l}\text { Time to onset } \\
\text { of symptoms }\end{array}$ & Symptom & Signs \\
\hline $\begin{array}{l}\text { VanDyke } \\
\text { (1983) [5] }\end{array}$ & $42 \mathrm{M}$ & Unwell for 10 days & Days & $P$ & Exudate (malignant) & $680 \mathrm{mls}$ & Minutes & Dyspnoea & LVF \\
\hline $\begin{array}{l}\text { Shenoy } \\
\text { (1984) [22] }\end{array}$ & $57 \mathrm{M}$ & Recent myocardial infarction & Days & $P$ & Transudate & $1000 \mathrm{mls}$ & Minutes & Dyspnoea & LVF \\
\hline $\begin{array}{l}\text { Glasser } \\
\text { (1988) [23] }\end{array}$ & $33 \mathrm{M}$ & $\begin{array}{l}\text { Respiratory tract infection } 3 \\
\text { months prior, history of Down's } \\
\text { and Ventricular Septal Defect }\end{array}$ & Weeks & S & Transudate & $2000 \mathrm{mls}$ & Minutes & Dyspnoea & LVF \\
\hline $\begin{array}{l}\text { Downey } \\
\text { (1991) [24] }\end{array}$ & $50 \mathrm{M}$ & $\begin{array}{l}\text { Traumatic ( } 3 \text { weeks post } \\
\text { motor vehicle accident) }\end{array}$ & Weeks & $P$ & Not specified & $\begin{array}{l}450 \mathrm{mls} \text { then } 1500 \\
\mathrm{mls}\end{array}$ & Minutes & Dyspnoea & LVF \\
\hline $\begin{array}{l}\text { Wolfe (1993) } \\
\text { [19] }\end{array}$ & $46 \mathrm{~F}$ & $\begin{array}{l}2 \text { weeks, history of } \\
\text { breast cancer prior }\end{array}$ & Weeks & $P$ & Exudate & $650 \mathrm{mls}$ & Weeks & Dyspnoea & LVF \\
\hline $\begin{array}{l}\text { Wolfe } \\
\text { (1993) [19] }\end{array}$ & $50 \mathrm{~F}$ & $\begin{array}{l}2 \text { weeks, history of } \\
\text { breast cancer prior }\end{array}$ & Weeks & $P$ & Exudate & $650 \mathrm{mls}$ & Weeks & Dyspnoea & LVF \\
\hline $\begin{array}{l}\text { Hamaya } \\
\text { (1993) [25] }\end{array}$ & $16 \mathrm{~F}$ & $\begin{array}{l}\text { Unwell, lymphoma with } \\
\text { pericardial effusion for } 3 \text { years }\end{array}$ & Months & $P$ & Not specified & $700 \mathrm{mls}$ & Weeks & Dyspnoea & $\begin{array}{l}\text { CS, and no } \\
\text { APO }\end{array}$ \\
\hline $\begin{array}{l}\text { Braverman } \\
\text { (1994) [26] }\end{array}$ & $27 \mathrm{~F}$ & $\begin{array}{l}\text { Unwell for } 3 \text { weeks } \\
\text { (Atrial Septal Defect closure } \\
13 \text { years prior) }\end{array}$ & Weeks & $P$ then $S$ & Transudate & $500 \mathrm{mls}$ then $100 \mathrm{mls}$ & Days & $\begin{array}{l}\text { Dyspnoea, pleuritic } \\
\text { chest pain }\end{array}$ & LVF, RVF, CS \\
\hline $\begin{array}{l}\text { Anguera } \\
\text { (1996) [27] }\end{array}$ & $68 \mathrm{~F}$ & $\begin{array}{l}\text { History of bowel cancer, } \\
\text { anorexia and dyspnoea for } \\
1 \text { month }\end{array}$ & Weeks & $P$ & Malignant & $800 \mathrm{mls}$ & Minutes & - & CS \\
\hline $\begin{array}{l}\text { Sunday } \\
\text { (1999) [8] }\end{array}$ & $60 \mathrm{~F}$ & $\begin{array}{l}3 \text { days of dyspnoea, lung cancer } \\
\text { with pericardial involvement }\end{array}$ & Days & S & Exudate & $700 \mathrm{mls}$ & Minutes & Dyspnoea & CS, LVF \\
\hline $\begin{array}{l}\text { Chamoun } \\
\text { (2003) [6] }\end{array}$ & $36 \mathrm{~F}$ & $\begin{array}{l}2 \text { months post Mitral valve } \\
\text { replacement and Tricuspid repair }\end{array}$ & Days & $P$ & Exudate & $1070 \mathrm{mls}$ & Hours & Dyspnoea & CS, LVF \\
\hline $\begin{array}{l}\text { Chamoun } \\
\text { (2003) [6] }\end{array}$ & $46 \mathrm{~F}$ & Metastatic cancer & Weeks & $P$ & Exudate & $1000 \mathrm{mls}$ & Hours & Dyspnoea & CS, LVF \\
\hline $\begin{array}{l}\text { Geffroy } \\
\text { (2004) [7] }\end{array}$ & $53 \mathrm{M}$ & $\begin{array}{l}1 \text { month post chemotherapy } \\
\text { for cancer }\end{array}$ & Weeks & S & Exudate & $1500 \mathrm{mls}$ & Not specified & Dyspnoea, hypoxia & $C S, L V F, R V F$ \\
\hline $\begin{array}{l}\text { Ligero } \\
(2006)[20]\end{array}$ & $41 \mathrm{~F}$ & $\begin{array}{l}\text { Lung cancer with hepatic } \\
\text { metastases }\end{array}$ & Days & $P$ & Exudate & $1000 \mathrm{mls}$ & Hours & Dyspnoea & LVF, RHF \\
\hline $\begin{array}{l}\text { Bernal } \\
(2007)[28]\end{array}$ & $45 \mathrm{~F}$ & Acute myeloid leukemia & Days & $P$ & Exudate & $500 \mathrm{mls}$ & Hours & Dyspnoea & CS, LVF \\
\hline $\begin{array}{l}\text { Dosios } \\
\text { (2007) [9] }\end{array}$ & $66 \mathrm{~F}$ & $\begin{array}{l}\text { Hematoma, } 10 \text { day history } \\
\text { of dyspnoea }\end{array}$ & Days & S & Exudate & 500 mls initially & Hours & - & CS \\
\hline $\begin{array}{l}\text { Sevimli } \\
\text { (2008) [17] }\end{array}$ & $42 \mathrm{~F}$ & $\begin{array}{l}\text { Infective - tuberculous } \\
\text { pericarditis }\end{array}$ & Days & S & Exudate & $500 \mathrm{mls}$ & Hours & Dyspnoea & CS and LVF \\
\hline $\begin{array}{l}\text { Khalili } \\
\text { (2008) [29] }\end{array}$ & $32 \mathrm{~F}$ & $\begin{array}{l}2 \text { months post aortic and mitral } \\
\text { valve replacement surgery }\end{array}$ & Weeks & $P$ & Transudate & $1000 \mathrm{mls}$ & Hours & Dyspnoea & CS \\
\hline
\end{tabular}


Table 1 Summary of reported cases of LVF post pericardiocentesis: Clinical characteristics (Continued)

\begin{tabular}{|c|c|c|c|c|c|c|c|c|c|}
\hline $\begin{array}{l}\text { Flores } \\
\text { (2009) [30] }\end{array}$ & $80 \mathrm{M}$ & $\begin{array}{l}\text { Unwell for weeks, multiple } \\
\text { myeloma, stent } 2 \text { weeks prior }\end{array}$ & Weeks & $P$ & Transudate & $1200 \mathrm{mls}$ & Days & Dyspnoea & CS and LVF \\
\hline $\begin{array}{l}\text { Karamichalis } \\
\text { (2009) [31] }\end{array}$ & $19 \mathrm{~F}$ & $\begin{array}{l}2 \text { months post motor vehicle } \\
\text { accident }\end{array}$ & Weeks & $P$ & Exudate & $1600 \mathrm{mls}$ & Hours & Dyspnoea & LVF \\
\hline $\begin{array}{l}\text { Lee } \\
\text { (2010) [18] }\end{array}$ & $14 \mathrm{M}$ & $\begin{array}{l}\text { Infective - tuberculous } \\
\text { pericarditis }\end{array}$ & Days & $P$ & Exudate & Not specified & Hours & Dyspnoea & CS, LVF \\
\hline $\begin{array}{l}\operatorname{Lim} \\
(2011)[32]\end{array}$ & $44 \mathrm{~F}$ & $\begin{array}{l}\text { Hypothyroidism related heart } \\
\text { failure. Dyspnoea and fatigue for } \\
4 \text { months }\end{array}$ & Weeks & S & Exudate & $1.3 \mathrm{~L}$ & $9 \mathrm{~h}$ & - & CS \\
\hline $\begin{array}{l}\text { Abdelsalam } \\
\text { (2012) [10] }\end{array}$ & $65 \mathrm{~F}$ & $\begin{array}{l}\text { Stage IV Non small cell lung } \\
\text { cancer for } 6 \text { months, } 1 \text { week of } \\
\text { dyspnoea }\end{array}$ & Weeks & $S$ & Malignant & $\begin{array}{l}\text { Complete drainage } \\
\text { of pericardial effusion } \\
\text { intraoperatively }\end{array}$ & Seconds & $\begin{array}{l}\text { Asystole during } \\
\text { surgery }\end{array}$ & CS \\
\hline $\begin{array}{l}\text { Weijers } \\
\text { (2013) [11] }\end{array}$ & $69 \mathrm{~F}$ & Weight loss and dyspnoea & - & $P$ & - & $800 \mathrm{mls}$ & $6 \mathrm{~h}$ & - & LVF \\
\hline $\begin{array}{l}\text { Liang } \\
\text { (2014) [1] }\end{array}$ & $56 \mathrm{~F}$ & $\begin{array}{l}\text { Polymyositis. Progressive } \\
\text { dyspnoea on exertion }\end{array}$ & - & $P$ & - & $\begin{array}{l}275 \mathrm{mls} \text { initially, with } \\
\text { ongoing drain }\end{array}$ & Several hours & Pleuritic chest pain & Nil \\
\hline $\begin{array}{l}\text { Versaci } \\
(2015)[16]\end{array}$ & $78 \mathrm{~F}$ & 3 months post mitral valve repair & Days & $P$ & Possibly transudate & $500 \mathrm{mls}$ & Hours & Dyspnoea & LVF \\
\hline
\end{tabular}

Abbreviations: $P$ percutaneous, $S$ surgical, CS cardiogenic shock (hypotension, tachycardia), LVF Left heart failure, RVF right heart failure 
Table 2 Summary of reported cases of LVF post pericardiocentesis: Electrocardiographic, biochemical, echocardiographic and outcome parameters

\begin{tabular}{|c|c|c|c|c|c|c|c|c|c|c|}
\hline Report & $\begin{array}{l}\text { LV function } \\
\text { pre tap }\end{array}$ & $\begin{array}{l}\text { LV function } \\
\text { post tap }\end{array}$ & $\begin{array}{l}\text { RV function post } \\
\text { tap }\end{array}$ & $\begin{array}{l}\text { Regional wall motion } \\
\text { abnormality }\end{array}$ & Bio marker & ECG & $\begin{array}{l}\text { Coronary artery } \\
\text { imaging }\end{array}$ & $\begin{array}{l}\text { Inotrope, IABP } \\
\text { or Intubation }\end{array}$ & Death & LV recovery \\
\hline $\begin{array}{l}\text { VanDyke } \\
\text { (1983) [5] }\end{array}$ & Normal & Normal (EF 67\%) & - & $\mathrm{Nil}$ & Normal & Normal & - & Intubation & No & Normal LV \\
\hline $\begin{array}{l}\text { Shenoy } \\
\text { (1984) [22] }\end{array}$ & - & $\begin{array}{l}\text { Mild LV } \\
\text { impairment }\end{array}$ & Normal & Septal hypokinesis & Normal & $\begin{array}{l}\text { T wave abnormality } \\
\text { and ST elevation V5-6 }\end{array}$ & - & - & No & $\begin{array}{l}\text { Normalised few } \\
\text { days later }\end{array}$ \\
\hline $\begin{array}{l}\text { Glasser } \\
\text { (1987) [23] }\end{array}$ & - & $\begin{array}{l}\text { Pulmonary } \\
\text { capillary wedge } \\
\text { pressure normal }\end{array}$ & $\begin{array}{l}\text { Normal (RVP } \\
\text { increased) }\end{array}$ & - & - & - & - & Intubation & No & $\begin{array}{l}\text { Clinical } \\
\text { improvement }\end{array}$ \\
\hline $\begin{array}{l}\text { Downey } \\
\text { (1991) [24] }\end{array}$ & - & $\begin{array}{l}\text { Inferred to be } \\
\text { normal }\end{array}$ & Normal & - & - & Normal & - & No & No & Normal LV \\
\hline $\begin{array}{l}\text { Wolfe } \\
\text { (1993) [19] }\end{array}$ & $\begin{array}{l}\text { Normal, } \\
E F>50 \%\end{array}$ & EF 30\% & - & Severe global hypokinesis of LV & - & - & - & - & No & $\begin{array}{l}\text { Normalised after } \\
7 \text { days }\end{array}$ \\
\hline $\begin{array}{l}\text { Wolfe } \\
\text { (1993) [19] }\end{array}$ & $\begin{array}{l}\text { Normal, } \\
E F>50 \%\end{array}$ & EF $25 \%$ & - & $\begin{array}{l}\text { Antero-apical akinesis and } \\
\text { apical dyskinesis }\end{array}$ & - & - & - & - & - & $\begin{array}{l}\text { Normalised after } \\
2 \text { weeks }\end{array}$ \\
\hline $\begin{array}{l}\text { Hamaya } \\
\text { (1993) [25] }\end{array}$ & Normal & - & - & Not provided & Normal & ST elevation & - & $\begin{array}{l}\text { Inotropes and } \\
\text { intubation }\end{array}$ & No & - \\
\hline $\begin{array}{l}\text { Braverman } \\
\text { (1994) [26] }\end{array}$ & EF $20 \%$ & EF $20 \%$ & $E F<15 \%$ & Not provided & - & - & - & - & - & $\begin{array}{l}\text { EF } 45 \% \text { in } 9 \text { days } \\
\text { then normalised } \\
\text { after a few weeks }\end{array}$ \\
\hline $\begin{array}{l}\text { Anguera } \\
\text { (1996) [27] }\end{array}$ & - & $\begin{array}{l}\text { Mildly impaired. } \\
\text { Normal capillary } \\
\text { wedge pressure }\end{array}$ & $\begin{array}{l}\text { Severely dilated and } \\
\text { severely impaired } \\
\text { contractility, EF } \\
<15 \%\end{array}$ & Paradoxical septal motion & - & - & $\begin{array}{l}\text { Normal } \\
\text { coronary } \\
\text { arteries }\end{array}$ & Inotropes & No & $\begin{array}{l}\text { Complete } \\
\text { recovery of } \\
\text { biventricular fn } \\
\text { after } 10 \text { days }\end{array}$ \\
\hline $\begin{array}{l}\text { Sunday } \\
\text { (1999) [8] }\end{array}$ & EF 65\% & EF $30 \%$ & $\begin{array}{l}\text { Severely impaired } \\
\text { contractility }\end{array}$ & Global hypokinesis & - & - & - & Intubation & Yes & No \\
\hline $\begin{array}{l}\text { Chamoun } \\
\text { (2003) [6] }\end{array}$ & $\begin{array}{l}\text { Normal, } \\
E F>50 \%\end{array}$ & EF $20 \%$ & - & $\begin{array}{l}\text { Regional wall motion } \\
\text { abnormality }\end{array}$ & - & SR & $\begin{array}{l}\text { Normal } \\
\text { coronary } \\
\text { arteries }\end{array}$ & $\begin{array}{l}\text { Inotropes and } \\
\text { IABP }\end{array}$ & No & $\begin{array}{l}\text { Normalised } \\
2 \text { weeks later }\end{array}$ \\
\hline $\begin{array}{l}\text { Chamoun } \\
\text { (2003) [6] }\end{array}$ & $\begin{array}{l}\text { Normal, } \\
E F>50 \%\end{array}$ & EF 20\% & - & $\begin{array}{l}\text { Akinesis of mid anterior wall } \\
\text { and septum /dilatation of LV }\end{array}$ & - & SR & - & No & No & $\begin{array}{l}\text { Normalised } \\
2 \text { weeks later }\end{array}$ \\
\hline $\begin{array}{l}\text { Geffroy } \\
\text { (2004) [7] }\end{array}$ & $\begin{array}{l}\text { Normal, } \\
E F>50 \%\end{array}$ & $E F>50 \%$ & $E F<15 \%$ & Akinetic and dilated RV & Elevated & Old RBBB & $\begin{array}{l}\text { Normal } \\
\text { coronary } \\
\text { arteries }\end{array}$ & $\begin{array}{l}\text { Inotropes and } \\
\text { intubation }\end{array}$ & Yes & - \\
\hline $\begin{array}{l}\text { Ligero } \\
\text { (2006) [20] }\end{array}$ & $\begin{array}{l}\text { Normal, } \\
\text { EF } 75 \%\end{array}$ & EF $25 \%$ & Severe impairment & $\begin{array}{l}\text { Akinesis of anterior, septum } \\
\text { and apex }\end{array}$ & Normal CK & Normal & $\begin{array}{l}\text { Normal } \\
\text { coronary } \\
\text { arteries }\end{array}$ & Inotropes & No & $\begin{array}{l}\text { Normalised } \\
10 \text { days later }\end{array}$ \\
\hline $\begin{array}{l}\text { Bernal } \\
(2007)[28]\end{array}$ & $\begin{array}{l}\text { Normal, EF } \\
60-65 \%\end{array}$ & EF $30 \%$ & - & $\begin{array}{l}\text { Akinesis of mid anterior wall, } \\
\text { anteroseptal akinesis with } \\
\text { apical sparing }\end{array}$ & Elevated & Sinus tachycardia & $\begin{array}{l}\text { CMR: no } \\
\text { myocardial } \\
\text { infarction }\end{array}$ & $\begin{array}{l}\text { Inotropes and } \\
\text { intubation }\end{array}$ & No & $\begin{array}{l}\text { Normalised } \\
1 \text { weeks later }\end{array}$ \\
\hline \multirow[t]{2}{*}{$\begin{array}{l}\text { Dosios } \\
\text { (2007) [9] }\end{array}$} & Normal LV fn & EF 25\% & $\begin{array}{l}\text { Moderately dilated, } \\
\text { impaired }\end{array}$ & Global hypokinesis & Elevated & - & - & $\begin{array}{l}\text { Inotropes and } \\
\text { intubation }\end{array}$ & Yes & - \\
\hline & & EF 20\% & - & & - & & & No & No & \\
\hline
\end{tabular}


Table 2 Summary of reported cases of LVF post pericardiocentesis: Electrocardiographic, biochemical, echocardiographic and outcome parameters (Continued)

\begin{tabular}{|c|c|c|c|c|c|c|c|c|c|c|}
\hline $\begin{array}{l}\text { Sevimli } \\
\text { (2008) [17] }\end{array}$ & $\begin{array}{l}\text { Normal, } \\
E F>50 \%\end{array}$ & & & $\begin{array}{l}\text { Akinesis in the left ventricular } \\
\text { apex, and severe hypokinesis in } \\
\text { the septum }\end{array}$ & & $\begin{array}{l}\text { Precordial TWI, } \\
\text { normalised later }\end{array}$ & $\begin{array}{l}\text { Normal } \\
\text { coronary } \\
\text { arteries }\end{array}$ & & & $\begin{array}{l}\text { Normalised } \\
10 \text { days later }\end{array}$ \\
\hline $\begin{array}{l}\text { Khalili } \\
\text { (2008) [27] }\end{array}$ & EF 35\% & $<10 \%$ & $E F<15 \%$ & Global hypokinesis & - & Widening of QRS & - & $\begin{array}{l}\text { Inotropes and } \\
\text { IABP- }\end{array}$ & Yes & - \\
\hline $\begin{array}{l}\text { Flores } \\
\text { (2009) [28] }\end{array}$ & EF 60\% & $13 \%$ & - & Global hypokinesis & Normal & Normal & $\begin{array}{l}\text { Old RCA } \\
\text { Branch lesion }\end{array}$ & Inotropes & No & $\begin{array}{l}\text { Normalised } \\
10 \text { days later }\end{array}$ \\
\hline $\begin{array}{l}\text { Karamichalis } \\
\text { (2009) [31] }\end{array}$ & - & - & - & & - & Bradycardia & - & $\begin{array}{l}\text { Inotropes and } \\
\text { tracheostomy }\end{array}$ & Yes & - \\
\hline $\begin{array}{l}\text { Lee } \\
\text { (2010) [18] }\end{array}$ & - & EF $20-30 \%$ & - & $\begin{array}{l}\text { Typical features of Takotsubo's } \\
\text { (diagnosed as such) }\end{array}$ & - & $\begin{array}{l}\text { Precordial TWI, } \\
\text { normalised later }\end{array}$ & $\begin{array}{l}\text { Normal } \\
\text { coronary } \\
\text { arteries }\end{array}$ & No & Yes & No \\
\hline $\begin{array}{l}\operatorname{Lim} \\
(2011)[32]\end{array}$ & $\begin{array}{l}\text { EF normal, } \\
73 \%\end{array}$ & EF $46 \%$ & - & $\begin{array}{l}\text { Segmental wall motion } \\
\text { abnormality }\end{array}$ & - & - & - & $\begin{array}{l}\text { Inotropes and } \\
\text { IABP }\end{array}$ & Yes & - \\
\hline $\begin{array}{l}\text { Abdelsalam } \\
(2012)[10]\end{array}$ & Vigorous & EF $10-15 \%$ & $\begin{array}{l}\text { Dilated and } \\
\text { impaired fn }\end{array}$ & Takotsubo pattern of akinesia & - & ST elevation & - & $\begin{array}{l}\text { Inotropes and } \\
\text { IABP }\end{array}$ & Yes & - \\
\hline $\begin{array}{l}\text { Weijers } \\
\text { (2013) [11] }\end{array}$ & Normal & Poor LV fn & - & $\begin{array}{l}\text { General hypokinesia and } \\
\text { anterior and septal akinesia }\end{array}$ & Normal & $\begin{array}{l}\text { TWI and Q waves in } \\
\text { anterolateral lead }\end{array}$ & - & - & No & $\begin{array}{l}\text { Complete } \\
\text { recovery of LV fn } \\
\text { several months } \\
\text { later }\end{array}$ \\
\hline $\begin{array}{l}\text { Liang } \\
(2014)[1]\end{array}$ & $\begin{array}{l}\text { Normal, } \\
\text { EF } 69 \%\end{array}$ & EF 39\% (on MRI) & Impaired & $\begin{array}{l}\text { Severe mid and apical } \\
\text { hypokinesis of both Ventricles } \\
\text { (diagnosis: Takotsubo's } \\
\text { cardiomyopathy) }\end{array}$ & - & - & $\begin{array}{l}\text { Normal } \\
\text { coronary } \\
\text { arteries }\end{array}$ & - & No & $\begin{array}{l}\text { LV normalised } \\
1 \text { week later }\end{array}$ \\
\hline $\begin{array}{l}\text { Versaci } \\
(2015)[16]\end{array}$ & $\begin{array}{l}\text { Normal, } \\
E F>50 \%\end{array}$ & EF $28 \%$ & - & $\begin{array}{l}\text { LV ballooning, typical feature of } \\
\text { Takotsubo's cardiomyopathy }\end{array}$ & Elevated & $\begin{array}{l}\text { QS wave in } \mathrm{V} 1-\mathrm{V} 4 \\
\text { with negative T wave } \\
\text { and ST elevation in } \\
\text { V5-V6 }\end{array}$ & $\begin{array}{l}\text { Normal } \\
\text { coronary } \\
\text { arteries }\end{array}$ & No & No & $\begin{array}{l}\text { Normalised after } \\
10 \text { days }\end{array}$ \\
\hline
\end{tabular}


$\beta$-adrenoceptor - mediated myocardial stunning in the apex [14]. These three pathophysiologic pathways are thought to contribute to the ischaemia, morphologic features and potential haemodynamic sequelae that can be seen in SCM.

More recent case reports have made reference to LV apical ballooning related to PDS as similar to SCM $[10,11,15-17]$, and have postulated the physiological stressor being cardiac tamponade along with emotional stress [16]. It is therefore possible that the transient ventricular systolic dysfunction in PDS is actually a variant form of stress cardiomyopathy. We carefully reviewed 25 cases of heart failure post pericardiocentesis in the literature (Tables 1 and 2), and we believe that seven cases (two considered to be SCM $[1,18]$ by the authors and five classified as PDS $[10,16,17,19,20]$ ) could be considered to have echocardiographic features of SCM.

SCM has relatively characteristic clinical presentation, with rise of cardiac enzymes [21], and often associated with ischaemic ECG changes (up to $44 \%$ of those with SCM have T-wave inversion and $41 \%$ ST elevation $[13,21])$. The clinical manifestations in PDS are more variable, ranging from asymptomatic in some to severe low cardiac output states in others. The primary clinical symptom in PDS has been reported as dyspnoea (Table 1). This is in contrast to chest pain being predominant in SCM (69-83 \% of presentations) $[13,21]$. In the majority of cases of PDS in the literature (Table 2) there was no cardiac enzyme rise, and ischaemic type changes on ECG were seen in a minority (seven of twenty five cases). In all the cases where ischaemic ECG changes where present except for one, there was concomitant apical and peri-apical regional wall motion abnormality, which could be classified as SCM also.

Generally SCM has a benign course, with recovery of LV function and good prognosis [12], whilst PDS has poorer outcomes and increased mortality [4]. Reports of PDS suggested normalization of LV dysfunction in 12 of 25 cases classified as PDS. Of the 12 cases that did recover LV function, four had LV impairment with classic SCM pattern of LV impairment on echocardiogram $[16,17,19,20]$. The normalisation of LV function in our patient 2 weeks subsequently is more in keeping with SCM.

Current literature has not specifically addressed risk factors for the development of ventricular dysfunction after pericardiocentesis. In our patient, the malignant nature of the effusion, the presence of tamponade and larger size of pericardial effusion [4], may have increased his predisposition to develop ventricular dysfunction. Amount and rate of fluid removed on initial decompression are also associated with development PDS $[4,5]$, however there are no guidelines regarding the maximum amount of pericardial fluid that can be drained immediately. There is consensus to stop initial drainage with improvement of symptoms or hemodynamic parameters, followed by gradual decompression through indwelling catheter [5].

Our patient's apical systolic dysfunction post pericardiocentesis was associated with chest discomfort, transient loss of $\mathrm{R}$ waves and rise in cardiac enzymes are typical of classic SCM. The clinical sequence of HR and $R R$ improving immediately post decompression and then increasing again hours after the procedure, was a useful clinical marker of myocardial dysfunction, prompting investigation which identified new ventricular impairment. It is likely that the frequency of transient LV dysfunction is underestimated in these patients.

\section{Conclusion}

We report a case of transient biventricular dysfunction post pericardiocentesis, with classic features of SCM. LV dysfunction post pericardiocentesis and in PDS is more prevalent than previously thought, and some previous reports of PDS may also be potentially considered as SCM complicating pericardiocentesis. In addition to judicious and gradual decompression to avoid ventricular dysfunction or PDS, patients undergoing therapeutic pericardiocentesis should have careful haemodynamic monitoring, as changes in parameters such as heart rate and respiratory rate can raise suspicion of acute LV impairment.

\section{Consent}

Written informed consent was unable to be obtained from the patient for publication of this Case report and any accompanying images, as he has passed away. His next of kin are not contactable after their subsequent return to their home country of China. Professor L. Kritharides, Head of Department, approves the publication of this report, with all patient identifiers kept confidential and material presented solely for educational purposes arising from the clinical encounter.

\section{Additional files}

Additional file 1: Video 1. Apical four chamber view demonstrating large pericardial effusion on presentation, with tamponade and diastolic compression of right heart chambers. Note the LV function is normal.

Additional file 2: Video 2. Parasternal long view demonstrating large pericardial effusion on presentation, with tamponade and diastolic compression of right heart chambers. Note the LV function is normal.

Additional file 3: Video 3. Apical four chamber view performed on the following morning after the patient experienced chest discomfort. The main finding is severe biventricular impairment, with akinesis of the apex and periapical areas. There is small residual pericardial effusion.

Additional file 4: Video 4. Parasternal long view performed on the following morning after the patient experienced chest discomfort. The main finding is severe biventricular impairment, with akinesis of the apex and periapical areas. There is small residual pericardial effusion. 
Additional file 5: Video 5. Apical four chamber view performed nearly 2 weeks after the pericardiocentesis and subsequent stress cardiomyopathy. They show normalisation of both left and right ventricular function.

Additional file 6: Video 6. Parasternal long view performed nearly 2 weeks after the pericardiocentesis and subsequent stress cardiomyopathy. They show normalisation of both left and right ventricular function.

\section{Abbreviations}

SCM: Stress Cardiomyopathy; LV: Left ventricle; RV: Right ventricle; PDS: Pericardial decompression syndrome; APO: Acute pulmonary oedema; ECG: Electrocardiogram; TTE: Transthoracic echocardiography; bpm: Beats per minute; HR: Heart rate; RR: Respiratory rate.

\section{Competing interests}

The authors declare that they have no competing interests.

\section{Authors' contributions}

All authors have approved the final article, contributed to conception, literature review, analysis and interpretation of the material, to the drafting of the manuscript and its critical revision; All authors agree to be accountable for all aspects of the work in ensuring that questions related to the accuracy or integrity of any part of the work are appropriately investigated and resolved.

Received: 9 April 2015 Accepted: 30 June 2015

Published online: 16 July 2015

\section{References}

1. Liang JJ et al. Apical ballooning syndrome in polymyositis following placement of pericardial drainage catheter. J Cardiovasc Dis. 2014;2:1.

2. Angouras DC, Dosios T. Pericardial decompression syndrome: a term for a well-defined but rather underreported complication of pericardial drainage. Ann Thorac Surg. 2010;89:1702-3. author reply 1703.

3. Dosios T, Theakos N, Angouras D, Asimacopoulos P. Risk factors affecting the survival of patients with pericardial effusion submitted to subxiphoid pericardiostomy. Chest. 2003;124:242-6.

4. Wagner PL, McAleer E, Stillwell E, et al. Pericardial effusions in the cancer population: prognostic factors after pericardial window and the impact of paradoxical hemodynamic instability. J Thorac Cardiovasc Surg. 2011;141:34-8

5. Vandyke Jr WH, Cure J, Chakko CS, Gheorghiade M. Pulmonary edema after pericardiocentesis for cardiac tamponade. N Engl J Med. 1983;309:595-6.

6. Chamoun A, Cenz R, Mager A, et al. Acute left ventricular failure after large volume pericardiocentesis. Clin Cardiol. 2003;26:588-90.

7. Geffroy A, Beloeil H, Bouvier E, Chaumeil A, Albaladejo P, Marty J. Prolonged right ventricular failure after relief of cardiac tamponade. Can J Anaesth. 2004;51:482-5

8. Sunday R, Robinson LA, Bosek V. Low cardiac output complicating pericardiectomy for pericardial tamponade. Ann Thorac Surg. 1999;67:228-31.

9. Dosios T, Stefanidis A, Chatziantoniou C, Sgouropoulou S. Thorough clinical investigation of low cardiac output syndrome after subxiphoid pericardiostomy. Angiology. 2007;58:483-6.

10. Abdelsalam M, Moritz TA, Snyder JA, Cheriyath P, Spizzieri CL. Paradoxical hemodynamic instability complicating pericardial window surgery for cardiac tamponade in a cancer patient. Tex Heart Inst J. 2012;39:711-3.

11. Weijers RW, Post JC. Transient left ventricular systolic dysfunction mimicking myocardial infarction after pericardiocentesis. Neth Heart J. 2013;21:364-6.

12. Pilgrim TM, Wyss TR. Takotsubo cardiomyopathy or transient left ventricular apical ballooning syndrome: A systematic review. Int J Cardiol. 2008;124:283-92.

13. Singh NK, Rumman S, Mikell FL, Nallamothu N, Rangaswamy C. Stress cardiomyopathy: clinical and ventriculographic characteristics in 107 North American subjects. Int J Cardiol. 2010;141:297-303.

14. Akashi YJ, Nef HM, Lyon AR. Epidemiology and pathophysiology of Takotsubo syndrome. Nat Rev Cardiol. 2015;12:387-9.

15. Yeh RW, Yu PB, Drachman DE. Takotsubo cardiomyopathy complicated by cardiac tamponade: classic hemodynamic findings with a new disease. Circulation. 2010;122:1239-41.

16. Versaci F, Donati R, Mezzanotte R, Chiariello L, Ammirati F. An unusual complication following pericardiocentesis: reversible left ventricular dysfunction. J Cardiovasc Med. 2015;16 Suppl 2:S133-5.
17. Sevimli S, Arslan S, Gundogdu F, Senocak H. Development of left ventricular apical akinesis and thrombus during pericardiocentesis for pericardial tamponade. Turk Kardiyoloji Dernegi arsivi: Turk Kardiyoloji Derneginin yayin organidir. 2008;36:338-41.

18. Lee SY, Lee SE, Choi JW, Choi SI, Chun EJ, Choi JY. A case of transient left ventricular apical ballooning syndrome in a child: clinical features and imaging findings. The international journal of cardiovascular imaging. 2010;26:345-51.

19. Wolfe MW, Edelman ER. Transient systolic dysfunction after relief of cardiac tamponade. Ann Intern Med. 1993;119:42-4.

20. Ligero C, Leta R, Bayes-Genis A. Transient biventricular dysfunction following pericardiocentesis. Eur J Heart Fail. 2006;8:102-4.

21. Samardhi H, Raffel OC, Savage M, et al. Takotsubo cardiomyopathy: an Australian single centre experience with medium term follow up. Internal Med J. 2012:42:35-42.

22. Shenoy MM, Dhar S, Gittin R, Sinha AK, Sabado M. Pulmonary edema following pericardiotomy for cardiac tamponade. Chest. 1984;86:647-8

23. Glasser F, Fein AM, Feinsilver SH, Cotton E, Niederman MS. Non-cardiogenic pulmonary edema after pericardial drainage for cardiac tamponade. Chest. 1988;94:869-70.

24. Downey RJ, Bessler M, Weissman C. Acute pulmonary edema following pericardiocentesis for chronic cardiac tamponade secondary to trauma. Crit Care Med. 1991;19:1323-5.

25. Hamaya Y, Dohi S, Ueda N, Akamatsu S. Severe circulatory collapse immediately after pericardiocentesis in a patient with chronic cardiac tamponade. Anesth Analg. 1993;77:1278-81.

26. Braverman AC, Sundaresan S. Cardiac tamponade and severe ventricular dysfunction. Ann Intern Med. 1994;120:442.

27. Anguera I, Pare C, Perez-Villa F. Severe right ventricular dysfunction following pericardiocentesis for cardiac tamponade. Int J Cardiol. 1997;59:212-4.

28. Bernal JM, Pradhan J, Li T, Tchokonte R, Afonso L. Acute pulmonary edema following pericardiocentesis for cardiac tamponade. Can J Cardiol. 2007;23:1155-6.

29. Khalili AA. Acute Heart Failure after Evacuation of Large Volume of Pericardial Effusion by Pericardiostomy. Res J Biooogical Sci. 2008:3(1):32-4

30. Flores VM et al. Transient left ventricular dysfunction following pericardiocentesis. An unusual complication to bear in mind. Rev Esp Cardiol. 2009;62(9):1071-2.

31. Karamichalis JM, Gursky A, Valaulikar G, Pate JW, Weiman DS. Acute pulmonary edema after pericardial drainage for cardiac tamponade. Ann Thorac Surg. 2009:88:675-7.

32. Lim AS, Paz-Pacheco E, Reyes M, Punzalan F. Pericardial decompression syndrome in a patient with hypothyroidism presenting as massive pericardial effusion: a case report and review of related literature. BMJ Case Rep. 2011;2011.

\section{Submit your next manuscript to BioMed Central and take full advantage of:}

- Convenient online submission

- Thorough peer review

- No space constraints or color figure charges

- Immediate publication on acceptance

- Inclusion in PubMed, CAS, Scopus and Google Scholar

- Research which is freely available for redistribution 